\title{
A PLANCHEREL FORMULA FOR PARABOLIC SUBGROUPS
}

\author{
MIE NAKATA
}

\begin{abstract}
We obtain explicit Plancherel formulas for the parabolic subgroups $P$ of $p$-adic unitary groups which fix one dimensional isotropic subspaces. By means of certain limits of difference operators (called strong derivatives), we construct a Dixmier-Pukanszky operator which compensates for the nonunimodularity of the group $P$.

Then, we compute the Plancherel formula of $N \cdot A$, where $N$ is the nilradical of $P$ and $A=Q_{p}^{\prime}$, the multiplicative group of nonzero $p$-adic numbers, by formulating a $p$-adic change of variable formula and using the strong derivative.
\end{abstract}

\section{INTRODUCTION}

Let $p$ be a prime number not equal to $2 . \mathbf{Q}_{p}$ denotes the field of $p$-adic numbers with $p$-adic norm $|\cdot|_{p}$.

In this paper we obtain the Plancherel formula of parabolic subgroups $P$ of $p$-adic unitary groups where the center of the nilradical has dimension one. To compensate for the nonunimodularity of the group we construct a pseudodifferential operator from a certain class of differential operators "strong derivatives" and the Plancherel formula turns out to take the form (Theorem 1.2)

$$
\begin{aligned}
f(1 P)= & c_{1} \int_{\widehat{S}_{1}} \operatorname{tr} \pi_{1, \sigma}(\widetilde{D} f) d \hat{\mu}_{S_{1}}(\sigma) \\
& +c_{2} \int_{\widehat{S}_{1}} \operatorname{tr} \pi_{n, \sigma}(\widetilde{D} f) d \hat{\mu}_{S_{2}}(\sigma) .
\end{aligned}
$$

We also compute the Plancherel formula (Theorem 3.1) of $N \cdot A$, where $N$ is the nilradical of $P$ and $A=\mathbf{Q}_{p}^{\prime}$, the multiplicative group of nonzero $p$-adic numbers, by formulating a $p$-adic change of variable formula (Proposition 2.4) and by using the string derivative

$$
\psi\left(1_{N \cdot A}\right)=T_{1}\left(\underline{D} \psi+p T_{p}(\underline{D} \psi)+T_{n}(\underline{D} \psi)+p T_{s p}(\underline{D} \psi)\right)
$$

where $T_{\lambda}(\psi)$ is the trace of an induced representation $\pi_{\lambda}(\psi)$.

Received by the editors April 29, 1987.

1980 Mathematics Subject Classification (1985 Revision). Primary 17B20, 22E35, 22E50, 43A32.

Key words and phrases. Parabolic subgroup, $p$-adic number field, Plancherel formula, DixmierPukanszky operator, and strong derivative. 


\section{Plancherel formula for a Parabolic subgroup $P_{E}$ OF $p$-ADIC UNITARY GROUPS WITH $\operatorname{dim} E=1$}

1. Preliminaries-strong derivative for a Dixmier-Pukanszky operator. For the Plancherel formula for nonunimodular groups, we define a Dixmier-Pukanszky operator to compensate for the nonunimodularity of the group. In the $p$-adic case, some power of the strong derivative plays a role.

First, we introduce the definition of the strong derivative and its important properties. We follow Onneweer's paper [16].

Let $\Gamma$ denote the character group $\widehat{\mathbf{Q}}_{p}$ of $\mathbf{Q}_{p}$. It contains a distinguished character $\chi_{0}$ such that $\left.\chi_{0}\right|_{B(0,0)}=1$ and $\left.\chi_{0}\right|_{B(0,-1)} \neq 1$, where $B(\alpha, n)$ is the ball centered at $\alpha$ with radius $1 / p^{n}$. Then $\Gamma=\left\{\chi_{y}: y \in \mathbf{Q}_{p}\right\}$ where $\chi_{y}(x)=\chi_{0}(y z)$ for all $x \in \mathbf{Q}_{p}$. By $d t$ we denote Haar measure on $\mathbf{Q}_{p}$, normalized so that the measure $B(0,0)$ is one. We mean also by $\|\chi\|$ the usual norm $|y|_{p}$ of $y$ if $\chi=\chi_{y}$.

Definition 1.1. Let $L_{\text {loc }}^{1}\left(\mathbf{Q}_{p}\right)=\left\{f: \mathbf{Q}_{p} \rightarrow C \mid f\right.$ is locally $\left.L^{1}\right\}$. For $m=$ $1,2,3, \ldots$, define $E_{m} f: \mathbf{Q}_{p} \rightarrow \mathbf{C}$ by

$$
E_{m} f(x)=\sum_{l=-\infty}^{m-1}\left(p^{l+1}-p^{l}\right)\left(f(x)-\Delta_{t} * f(x)\right)
$$

where

$$
\Delta_{l}(x)= \begin{cases}p^{l} & \text { for } x \in B(0, l) \\ 0 & \text { otherwise }\end{cases}
$$

and $\Delta_{l} * f(x)=p^{l} \int_{B(x, l)} f(t) d t$ is the convolution which is the average of $f$ over $B(x, l)$.

If $\lim _{m \rightarrow \infty} E_{m} f(x)$ exists for $x \in \mathbf{Q}_{p}$, then this limit is called the pointwise derivative of $f$ at $x$ and is denoted by $f^{|1|}(x)$.

If $f \in C\left(\mathbf{Q}_{p}\right),\left\{q: \mathbf{Q}_{p} \rightarrow \mathbf{C} \mid g\right.$ is continuous $\}$, and if the limit is in $C\left(\mathbf{Q}_{p}\right)$, then the limit is called the strong derivative of $f$ in $C\left(\mathbf{Q}_{p}\right)$ and is denoted by $D^{|1|} f$.

Remark. It can be checked that locally constant functions with compact support have strong derivatives.

The following theorem gives an important property of the strong derivative.

Theorem 1.1 (Onneweer [16]). (1) If $\chi \in \Gamma$ and $x \in \mathbf{Q}_{p}$, then $\chi^{|1|}$ exists and $\chi^{|1|}(x)=\|\chi\|(x)$.

(2) Every $\chi \in \Gamma$ is strongly differentiable in $C\left(\mathbf{Q}_{p}\right)$, the space of all continuous functions.

(3) For $f \in L^{1}\left(\mathbf{Q}_{p}\right)$ and $n=1,2,3, \ldots$,

$$
\left(E_{n} f\right)^{\wedge}(\chi)= \begin{cases}\|\chi\| \hat{f}(\chi) & \text { if } \chi \in \Gamma_{n}, \\ p^{n} \hat{f}(\chi) & \text { if } \chi \notin \Gamma_{n},\end{cases}
$$


where $\Gamma_{n}=\left\{\chi=\chi_{y} \in \Gamma \mid y \in B(0,-n)\right\}$ and ${ }^{\wedge}$ is the Fourier transform defined by $\hat{f}(\chi)=\int_{\mathbf{Q}_{p}} f(t) \overline{\chi(t)} d t$.

Corollary (Onneweer [16]). Let $D\left(D^{|1|}\right)$ denote the domain of $D^{|1|}$. Then for $f \in D\left(D^{|1|}\right),\left(D^{|1|} f\right)^{\wedge}(\chi)=\|\chi\| \hat{f}(\chi)$, for all $x \in \Gamma$.

This property implies that the strong derivative can be characterized as a multiplication operator $M_{|y|_{p}}$ by $|y|_{p}$ via Fourier transform, i.e. $D^{|1|}=\wedge^{-1}$. $M_{|y|_{p}} \cdot \wedge$. Clearly, $D^{|1|}$ is positive, selfadjoint, invertible, since $|y|_{p}$ is positive, real-valued, and takes zero only on a set of measure zero.

2. Plancherel formula for parabolic subgroups $P_{e}$ of $p$-adic unitary groups with $\operatorname{dim} E=1$. Let $p$ be a rational prime number not equal to 2 and let $\mathbf{Q}_{p}$ denote the $p$-adic number field. Let $(W, h)$ be a Hermitian vector space over $\widetilde{\mathbf{Q}}_{p}$ relative to $\mapsto \bar{a}$. Here $\widetilde{\mathbf{Q}}_{p}=\mathbf{Q}_{p}(\sqrt{p})$ is a quadratic extension by $\sqrt{p}$, $a \mapsto \bar{a}$ is the nontrivial element of the Galois group of $\widetilde{\mathbf{Q}}_{p}$ over $\mathbf{Q}_{p}$.

Let $E \subseteq W$ be an isotropic subspace, i.e. $h(E, E)=\{0\}$. It is known that there is a totally isotropic subspace $E^{\prime} \subseteq W$ such that $\operatorname{dim} E=\operatorname{dim} E^{\prime}$ and $h$ is nondegenerate. We write $W=V+E^{\prime}+E$ so that $V=\left(E^{\prime}+E\right)^{\perp}$ relative to $h$. If we denote by $U(W, h)$ the group of unitary operators on $W$ with respect to the Hermitian form $h$, then $P_{E}=\{b \in U(W, h) \mid b E \subseteq E\}$ is a maximal parabolic subgroup of $U(W, h)$ and this construction gives all such maximal parabolics.

The structure of such $p$-adic parabolic subgroups is essentially the same as in the real case; for $n=\operatorname{dim} V$ and $S=\operatorname{dim} E$ the following is true:

$$
P_{E}=(Z \cdot X) \cdot\left(G L(E) \times U\left(V,\left.h\right|_{V \times V}\right)\right)
$$

where $N=Z \cdot X$ is the nilradical, $L=G L(E) \times U\left(V,\left.h\right|_{V \times V}\right)$ is the Levi component, and $Z=\left\{z \in \mathbf{Q}_{p}^{s \times s}\right.$, an $s \times s$ matrix: $\left.{ }^{t} \bar{z}=-z\right\}, X=\mathbf{Q}_{p}^{s \times n}=\{s \times n$ matrices over $\left.\widetilde{\mathbf{Q}}_{p}\right\}$. The group multiplication of $P$ is

$$
\begin{aligned}
& \left(z_{1}, x_{1}, \gamma_{1}, g_{1}\right)\left(z_{2}, x_{2}, \gamma_{2}, g_{2}\right) \\
& \quad\left(z_{1}+\gamma_{1} z_{2}{ }^{t} \bar{\gamma}_{1}+\operatorname{Im} H\left(x_{1}, \gamma_{1} x_{2} g_{1}^{-1}\right), x_{1}+\gamma_{2} x_{2} g_{1}^{-1}, \gamma_{1} \gamma_{2}, g_{1} g_{2}\right)
\end{aligned}
$$

where $H\left(x^{\prime}, x^{\prime \prime}\right)=x^{\prime \prime} M^{-1 t} \bar{x}^{\prime}$ and $\operatorname{Im} H\left(x^{\prime}, x^{\prime \prime}\right)=\frac{1}{2}\left[H\left(x^{\prime}, x^{\prime \prime}\right)-H\left(x^{\prime \prime}, x^{\prime}\right)\right]$. By choosing a basis $\left\{v_{1}, \ldots, v_{n}\right\}$ of $V,\left\{e_{1}^{\prime}, \ldots, e_{s}^{\prime}\right\}$ of $E^{\prime}$ and $\left\{e_{1}, \ldots, e_{s}\right\}$ of $E$ such that $h\left(e_{i}^{\prime}, e_{j}\right)=\delta_{i j}$, the matrix form of $M_{h}$ of the Hermitian form $h$ is written as

$$
M_{h}=\left(\begin{array}{ccc}
M & 0 & 0 \\
0 & 0 & I \\
0 & I & 0
\end{array}\right) \quad \text { with }{ }^{t} \bar{M}=M .
$$

The following theorem can be proved in essentially the same way as the real case (Lipsman-Wolf [11]). 
Theorem 1.2. Let $P=P_{E}$ with $\operatorname{dim} E=1$. Then

$$
f\left(1_{P}\right)=c_{1} \int_{\widehat{S}_{1}} \operatorname{tr} \pi_{1, \sigma}(\widetilde{D} f) d \hat{\mu}_{S_{1}}(\sigma)+c_{2} \int_{\widehat{S}_{2}} \operatorname{tr} \pi_{n, \sigma}(\widetilde{D} f) d \hat{\mu}_{S_{2}}(\sigma),
$$

where $c_{i}$ are some real constants, $S_{i}=U\left(\widetilde{\mathbf{Q}}_{p}, h_{i}\right) \times U\left(V,\left.h\right|_{V \times V}\right), i=1,2, h_{1}$ and $h_{2}$ are inequivalent Hermitian forms over $\widetilde{\mathbf{Q}}_{p}$, for example, $h_{1}=(1)$ and $h_{2}=(\mu)$ where $\mu$ is a unit element of $\mathbf{Q}_{p}$ which is not a square as an element of residue class field, $\hat{\mu}_{S_{1}}$ is the Plancherel measure on $\hat{S}_{i}$ corresponding to a uniquely determined Haar measure $\mu_{S_{i}}$ according to the formula

$$
\int_{L} f(x) d x=\int_{S_{i} \backslash L} \int_{S_{i}} f\left(s_{i} x\right) d s_{i} d \bar{x}
$$

where $d \bar{x}$ is the Borel measure on $S_{i} \backslash L$ and $\pi_{1, \sigma}=\operatorname{Ind}_{N S_{1}}^{P}\left(\tilde{\gamma}_{1} \times \sigma\right), \sigma \in \widehat{S}_{1}$, $\pi_{n, \sigma}=\operatorname{Ind}_{N S_{2}}^{P}\left(\tilde{\gamma}_{n} \times \sigma\right), \sigma \in \widehat{S}_{2}, \tilde{\gamma}_{1}$ and $\tilde{\gamma}_{2}$ are extensions of $\gamma_{1}$ and $\gamma_{2}$, representatives of L-orbits of $\widehat{N}$. Finally, $\widetilde{D}=D^{n+1}$, where $D=|1|$, the $(n+1)$ th power of the strong derivative.

Now, to obtain a more explicit formula for our parabolic subgroup $P$, we have to compute $p$-adic $L$-orbits of $Z$, which will turn out to be equivalent to classifying nondegenerate Hermitian forms over $\widetilde{\mathbf{Q}}_{p}$, and we have to compute the corresponding stability groups. We treat this without any restrictions on $\operatorname{dim} E$.

3. $p$-adic $L$-orbits of $Z$ and the $L$-stabilizer. In order to apply Mackey's little group method, we first find the $L$-orbits on $Z$.

$L$ acts on $Z$ by $(\gamma, g) \cdot z=\gamma z \gamma^{s}$. So the $L$-orbits are represented by $\left\{\sqrt{p} h \mid[h]\right.$ is an equivalence class of Hermitian forms on $\left.\widetilde{\mathbf{Q}}_{p}^{s}\right\}$. Thus we are going to classify Hermitian forms on $\widetilde{\mathbf{Q}}_{p}^{s}$ up to equivalence.

The following lemma can be proved easily.

Lemma 1.1. Given a symmetric bilinear form $q$ on $\mathbf{Q}_{p}^{2 s}$, there is a Hermitian form $h$ on $\widetilde{\mathbf{Q}}_{p}^{s}$ such that

$$
h(X, Y)=q(\widetilde{X}, \widetilde{Y})+\frac{1}{\sqrt{p}} q(\sqrt{p} \widetilde{X}, \widetilde{Y})
$$

if and only if $q(\sqrt{p} \tilde{X}, \tilde{Y})+q(\widetilde{X}, \sqrt{p}, \widetilde{Y})=0$ for all $X, Y \in \overline{\mathbf{Q}}_{p}^{s}$, where $\widetilde{X}$ and $\tilde{Y}$ are elements of $\mathbf{Q}_{p}^{2 s}$ corresponding to $X$ and $Y$ under the isomorphism $\mathbf{Q}_{p}^{2 s} \cong$ $\mathbf{Q}_{p}(\sqrt{p})^{s}$ and $\sqrt{p} \widetilde{X}$ is regarded as an operator $\widetilde{X} \mapsto \sqrt{p} \widetilde{X}$ on $\mathbf{Q}_{p}^{2 s}$ operating on $\widetilde{X}$.

Thus we only need to find the symmetric nondegenerate bilinear forms on $\widetilde{\mathbf{Q}}_{p}^{s}$, up to equivalence, satisfying the condition of Lemma 1.1 . 
Definition 1.2. A quadratic form $q$ on $V$ is said to be isotropic if $q(x)=0$ for some $x \neq 0$ in $V$ and is said to be an anisotropic otherwise.

Definition 1.3. A Hermitian form is said to be anisotropic if the quadratic form defined by the Hermitian form is anisotropic.

We are going to classify the anisotropic Hermitian forms first.

Lemma 1.2. (1) Anisotropic nondegenerate Hermitian forms over $\widetilde{\mathbf{Q}}_{p}$ are at most two dimensional.

(2) There are only two equivalence classes of 1-dimensional ones; they are represented by $1 \times 1$ matrices (1) and $(u)$, where $u \in \mathbf{Q}_{p}$ is a unit such that $u$, as an element of the residue class field, is not a square.

(3) There is only one equivalence class of 2-dimensional ones; it is represented by the $2 \times 2$ matrix $\left(\begin{array}{cc}1 & 0 \\ 0 & -u\end{array}\right)$.

Proof. By the theory of quadratic forms, we know that anisotropic nondegenerate quadratic forms are at most 4-dimensional (see [9]). Hence anisotropic nondegenerate Hermitian forms are at most 2-dimensional. So we get (1).

Let $q$ be a quadratic form which gives rise to a Hermitian form. Then $\operatorname{dim} q$ is either 2 or 4 . If $\operatorname{dim} q=2$, write $q=\left(\begin{array}{ll}a & 0 \\ 0 & b\end{array}\right)$ with $a, b \in \mathbf{Q}_{p}$. The condition in Lemma 1.1 that $q(\sqrt{p} \tilde{X}, \tilde{Y})+q(\tilde{X}, \sqrt{p}, \tilde{Y})=0$ for all $\tilde{X}, \tilde{Y} \in \mathbf{Q}_{p}^{2 s}$ means ${ }^{t} \sqrt{p} q+q \sqrt{p}=0$, where ${ }^{t} \sqrt{p}$ means the transpose of the operator $\sqrt{p}$. So

$$
\left(\begin{array}{ll}
0 & 1 \\
p & 0
\end{array}\right)\left(\begin{array}{ll}
a & 0 \\
0 & b
\end{array}\right)+\left(\begin{array}{ll}
a & 0 \\
0 & b
\end{array}\right)\left(\begin{array}{ll}
0 & p \\
1 & 0
\end{array}\right)=\left(\begin{array}{ll}
0 & 0 \\
0 & 0
\end{array}\right) .
$$

So $b=-a p$. Hence $q=\left(\begin{array}{cc}a & 0 \\ 0 & -a p\end{array}\right)$.

It is known that there are only six inequivalence classes of nondegenerate anisotropic quadratic forms over $\mathbf{Q}_{p}$, namely

$$
\begin{aligned}
& \left(\begin{array}{ll}
1 & \\
& -u
\end{array}\right), \quad\left(\begin{array}{ll}
p & \\
& -p u
\end{array}\right), \quad\left(\begin{array}{ll}
1 & \\
& -p
\end{array}\right), \\
& \left(\begin{array}{ll}
u & \\
& -p u
\end{array}\right), \quad\left(\begin{array}{ll}
1 & \\
& -p u
\end{array}\right), \quad\left(\begin{array}{ll}
u & \\
& -p
\end{array}\right) .
\end{aligned}
$$

We notice that among those only $\left(\begin{array}{ll}{ }^{1} & -p\end{array}\right)$ and $\left(\begin{array}{ll}u & -p u\end{array}\right)$ are of the form $\left(\begin{array}{cc}a & 0 \\ 0 & -a p\end{array}\right)$.

If $\operatorname{dim} q=4$, again from the theory of quadratic forms over $\mathbf{Q}_{p}$, we know that there is only one class of nondegenerate anisotropic quadratic forms, namely

$$
q=\left(\begin{array}{llll}
1 & & & \\
& -u & & \\
& & -p & \\
& & & -u p
\end{array}\right)
$$


Claim. The Hermitian form giving $q=\left({ }^{1}-p\right)$ is $h=(1)$. The Hermitian form giving $q^{\prime}=\left({ }^{u}{ }_{-u p}\right)$ is $h^{\prime}=(u)$. The Hermitian form giving

$$
q^{\prime \prime}=\left(\begin{array}{cccc}
1 & & & \\
& -u & & \\
& & -p & \\
& & & u p
\end{array}\right)
$$

is $h^{\prime \prime}=\left(\begin{array}{ll}1 & \\ & \end{array}\right)$.

Proof of Claim. Note $h(X, X)=q(\widetilde{X}, \widetilde{X})$ because the skew-symmetric part gives zero. Let $X=x+\sqrt{p} y, x, y \in \mathbf{Q}_{p}^{s}$. Then

$$
\begin{aligned}
h(X, X) & =\bar{X}(1) X=\bar{X} X=(x-\sqrt{p} y)(x+\sqrt{p} y)=(x, y)\left(\begin{array}{ll}
1 & \\
& -p
\end{array}\right)\left(\begin{array}{l}
x \\
y
\end{array}\right) \\
& =q((x, y),(x, y))=q(\widetilde{X}, \widetilde{X}) .
\end{aligned}
$$

So $h=(1)$ is the Hermitian form giving $q=\left({ }^{1}-p\right)$. Similarly, for $q^{\prime}=$ $\left(\begin{array}{ll}u & \\ & -u p\end{array}\right)$ and

$$
q^{\prime \prime}=\left(\begin{array}{llll}
1 & & & \\
& -u & & \\
& & -p & \\
& & & u p
\end{array}\right) \text {. }
$$

This proves the claim.

Next, we have to show the following: If $q$ and $q^{\prime}$ are given by Hermitian forms $h$ and $h^{\prime}$, respectively, and if $q$ and $q^{\prime}$ are not equivalent, then $h$ and $h^{\prime}$ are not equivalent.

To prove this suppose that $h$ and $h^{\prime}$ are equivalent. Then there exists $A \in G L\left(s, \widetilde{\mathbf{Q}}_{p}\right)$ such that $h^{\prime}(A X, A Y)=h(X, Y)$ for all $X, Y \in \widetilde{\mathbf{Q}}_{p}^{s}$. Now,

$$
h^{\prime}(A X, A Y)=q^{\prime}(\tilde{A} \widetilde{X}, \tilde{A} \widetilde{Y})+\frac{1}{\sqrt{p}} q^{\prime}(\tilde{A} \widetilde{X}, \tilde{A} \widetilde{Y}),
$$

where $\widetilde{A}$ is the operator $\in G L\left(2 s, \mathbf{Q}_{p}\right)$ determined by $A$. Also,

$$
h(x, Y)=q(\tilde{X}, \tilde{Y})+\frac{1}{\sqrt{p}} q(\sqrt{p} \tilde{X}, \tilde{Y}) .
$$

Comparing the $\mathbf{Q}_{p}$ components we get $q^{\prime}(\tilde{A} \tilde{X}, \tilde{A} \widetilde{Y})=q(\tilde{X}, \widetilde{Y})$. So $q$ and $q^{\prime}$ are inequivalent. Therefore now we know that the 1-dimensional case (1) and (u) are inequivalent.

Finally, we have to show that there are no more inequivalent classes.

We first consider the 1-dimensional case. Let $h=(1)$ and $h^{\prime}=(u)$. Suppose that there exists $h^{\prime}=(a)$ with $a \in \mathbf{Q}_{p}$ such that $h^{\prime \prime} \nsim h^{\prime}$ where $\sim$ means equivalent and $\nsim$ means not equivalent. This implies that there is no $\alpha \in$ $\mathbf{Q}_{p}(\sqrt{p})$ such that $\bar{\alpha} \alpha u=a$, i.e., $a \neq x^{2}-p y^{2}$ for any $x, y \in \mathbf{Q}_{p}$ and $a \neq u\left(x^{2}-p y^{2}\right)$ for any $x, y \in \mathbf{Q}_{p}$. Now, let $q^{\prime \prime}$ be the quadratic form given 
by $h^{\prime \prime}$. Then $q^{\prime \prime}=\left({ }^{a}{ }_{-a p}\right)$. Since $q^{\prime \prime}$ is anisotropic and nondegenerate, either $q^{\prime \prime} \sim\left(\begin{array}{ll}{ }^{1}{ }_{-p}\end{array}\right)$ or $q^{\prime \prime} \sim\left({ }^{u}-u p\right)$. If $q^{\prime \prime} \sim\left({ }^{1}-p\right)$, there must be $c \in G L\left(2, \mathbf{Q}_{p}\right)$ such that ${ }^{t} C\left(\begin{array}{ll}1 & -p\end{array}\right) C=\left(\begin{array}{ll}a & -a p\end{array}\right)$. Write $C=\left(\begin{array}{ll}x & y \\ z & w\end{array}\right)$. Then

$$
{ }^{t} C\left(\begin{array}{ll}
1 & \\
& -p
\end{array}\right) C=\left(\begin{array}{l}
x^{2}-p z^{2} x y-p z w \\
x y-p z w y^{2}-p w^{2}
\end{array}\right) \text {. }
$$

So ${ }^{t} C\left({ }^{1}-p\right) C=\left({ }^{a}-a p\right)$ forces that $x^{2}-p z^{2}=a$ in particular, which is impossible by the assumption that $h^{\prime \prime} \nsim h$. By a similar argument, there is no $h^{\prime \prime}$ such that $h^{\prime \prime} \nsim h^{\prime}$.

Now, to complete the classification we have to consider isotropic ones. We recall that if $h$ is a 2-dimensional nondegenerate isotropic Hermitian form over $\widetilde{\mathbf{Q}}_{p}$, then there exists a basis with respect to which $h$ has its matrix representation $\left(\begin{array}{cc}1 & 0 \\ 0 & -1\end{array}\right)$. $\widetilde{\mathbf{Q}}_{p}$.

Here is the result of classification of nondegenerate Hermitian forms over

Proposition 1.1. Any nondegenerate Hermitian form over $\widetilde{\mathbf{Q}}_{p}$ is of the form

$$
\left(\begin{array}{llllll}
1 & & & & & \\
& -1 & & & & \\
& & \ddots & & & \\
& & & 1 & & \\
& & & & -1 & \\
& & & & & \\
& & & & & A
\end{array}\right) \text { or }\left(\begin{array}{llllll}
1 & & & & & \\
& -1 & & & \\
& & \cdot & & & \\
& & & \cdot & & \\
& & & 1 & \\
& & & & -1
\end{array}\right)
$$

with $A$ anisotropic. Hence, the number of equivalence classes of s-dimensional nondegenerate Hermitian forms over $\widetilde{\mathbf{Q}}_{p}$ is always 2 .

In the 2-dimensional case, the argument is the same. So we omit this. Hence we have proved Lemma 1.2.

The translation of Proposition 1.1 over to a result on the $L$-orbits of $Z$ is as follows:

Proposition 1.2. For any positive integer $s$ there are precisely two generic $L$ orbits of $Z$; they are represented by $\sqrt{p} h_{1}, \sqrt{p} h_{2}$ where $h_{1}$ and $h_{2}$ are inequivalent nondegenerate Hermitian forms over $\widetilde{\mathbf{Q}}_{p}$. The L-stabilizer $S_{i}$ of $\sqrt{p} h_{i}$ is given by $S_{i}=U\left(\widetilde{\mathbf{Q}}_{p}^{2}, h_{i}\right) \times U\left(V,\left.h\right|_{V \times V}\right)$.

4. Dixmier-Pukanszky operator for $P_{E}$ with $\operatorname{dim} E=1$. We shall show that a certain power of the strong derivative will give a Dixmier-Pukanszky operator for a parabolic subgroup $P_{E}$ of $U(W, h)$ with $\operatorname{dim} E=1$.

For this parabolic group the modular function $\delta_{P}$ of $P$ is $\delta_{P}(z, x, a, m)=$ $|a|_{p}^{2(1+n)}$, where $A=\mathbf{Q}_{p}^{s}=$ nnonzero $p$-adic numbers $\}$, since the action of $A$ on $N$ is $a \cdot(z, x)=\left(a^{2} z, a x\right)$ and only $A$-part of $L$ has significance for $\delta_{P}$. 
Now, let $\widetilde{D}=D^{n+1}$ as in $\S 2$. Then this $\widetilde{D}$ clearly satisfies the criterion for being a Dixmier-Pukanszky operator, namely the criterion $x \cdot \widetilde{D}=\delta_{p}(x) \widetilde{D}$ for all $x \in L$ where $x \cdot \widetilde{D} f(y)=\widetilde{D} f\left(x y x^{-1}\right)$.

Hence $\widetilde{D}$ plays the role of Dixmier-Pukanszky operator and the proof of the theorem is complete.

\section{Plancherel formula for $N \cdot A$}

In this section we compute directly the Plancherel formula for $N \cdot A$.

Let $(W, h)$ be a Hermitian vector space over $\widetilde{\mathbf{Q}}_{p}$ relative to $a \mapsto \bar{a}$ and consider the case that the dimension of the center of $N$ is 1 as before. To make the comparison with the real case clearer, we choose $h$ so that

$$
M_{h}=\left(\begin{array}{ccc}
I & 0 & 0 \\
0 & 0 & I \\
0 & I & 0
\end{array}\right)
$$

Hence our group is $N \cdot A$ with $N=Z \cdot X, Z=\left\{\sqrt{p} z \mid z \in \mathbf{Q}_{p}\right\}, X=\widetilde{\mathbf{Q}}_{p}^{n}$, $A=\mathbf{Q}_{p}^{n}, A=\mathbf{Q}_{p}^{S}=$ the nonzero $p$-adic numbers and $p$ is a prime number not equal to 2 . We identify the group $N \cdot A$ with $\mathbf{Q}_{p} \times \mathbf{Q}_{p}^{n} \times \mathbf{Q}_{p}^{n} \times \mathbf{Q}_{p}^{S}$ with group multiplication:

$(z, y, x, a)\left(z^{\prime}, y^{\prime}, x^{\prime}, a^{\prime}\right)=\left(z+a^{2} z^{\prime}+a y^{\prime} x-a x^{\prime} y, y+a y^{\prime}, x+a x^{\prime}, a a^{\prime}\right)$.

The computation technique of the computation of the Plancherel formula for this particular group is mostly the same as the real case (see [4]). But in the $p$-adic case it is necessary to find a $p$-adic change of variable formula.

1. $p$-adic change of variable formula. A computation shows the following.

Proposition 2.1. Let $\alpha \in \mathbf{Q}_{p}$ and $n \in \mathbf{Z}$ be such that $0 \notin B(\alpha, n)$ where $B(\alpha, n)=\left\{x \in \mathbf{Q}_{p}|| \alpha-\left.x\right|_{p} \leq 1 / p^{n}\right\}$. Let $S: \mathbf{Q}_{p} \rightarrow \mathbf{Q}_{p}$ be the square function, i.e., $S(x)=x^{2}$. Then $S(B(\alpha, n))=B\left(\alpha^{2} n+k\right)$, where $|\alpha|_{p}=1 / p k$.

Now, the idea of the proof of the $p$-adic change of variable formula is that first we observe that the whole $\mathbf{Q}_{p}$ is a disjoint union of shells centered at 0 , i.e. Shell $(0, n)=\left\{\left.x \in \mathbf{Q}_{p}|| x\right|_{p}=1 / p^{n}\right\}$ and observe that each shell is a disjoint union of $p-1$ balls of radius $1 / p^{n+1}$. Then we obtain the formula on shells and eventually obtain the formula on $\mathbf{Q}_{p}$ by dominant convergence theorem.

The following lemma and the proposition are easily obtained by computation.

Lemma 2.1. The square function $S$ is one-one on $B(\alpha, n)$ if and only if $0 \notin$ $B(\alpha, n)$.

We need also the following technical proposition.

Proposition 2.2. For $p \neq 2$, let $B(\alpha, n) \subseteq \mathbf{Q}_{p}$ be such that $0 \notin B(\alpha, n)$. Then

$$
\int_{B(\alpha, n)} 1_{B(\beta, k)}\left(x^{2}\right)|x|_{p} d x=\int_{S(B(\alpha, n))} 1_{B(\beta, k)}(x) c x
$$


for any $B(\beta, k) \subseteq \mathbf{Q}_{p}$. Here $S: \mathbf{Q}_{p} \rightarrow \mathbf{Q}_{p}$ is defined by $S(x)=x^{2}$ as before and $1_{A}$ is a characteristic function on $A$, i.e.,

$$
1_{A}(x)= \begin{cases}1 & \text { if } x \in A \\ 0 & \text { otherwise }\end{cases}
$$

The next step is to obtain the formula on shells. We recall that each shell is a disjoint union of balls. We will make use of this fact fully later.

Lemma 2.2. Let $p \neq 2$.

$$
S\left(B\left(i p^{-n},-n+1\right)\right)=S\left(B\left((p-i) p^{-n}-n+1\right)\right)
$$

for all $i=1, \ldots,(p-1) / 2$. It can be proven by direct computation. Here $i$ is the intermediate form of the p-adic change of variable formula.

Proposition 2.3.

$$
\int_{\text {Shell }(0,0)} f\left(x^{2}\right)|x|_{p} d x=2 \int_{S(\operatorname{Shell}(0,0))} f(x) d x
$$

for all $f \in C_{c}^{\infty}\left(\mathbf{Q}_{p}\right)=\left\{f: \mathbf{Q}_{p} \rightarrow \mathbf{C} \mid f\right.$ is locally constant and has compact support $\}$, where $\operatorname{Shell}(0,0)=\left\{\left.x \in \mathbf{Q}_{p}|| x\right|_{p}=1\right\}$.

Proof. Note: We can write Shell $(0,0)$ as the disjoint union of balls, Shell $(0,0)$ $=B(1,1) \cup \cdots \cup B(p-1,1)$. Also, since $f$ is compactly supported, supp $f \subseteq$ $B(0,-n)$ for some $n \in \mathbf{Z}$ and since $f$ is locally constant, $f$ is constant on balls of radius $1 / p^{k}$ for some $k \in \mathbf{Z}$. We may assume that $n>-k$. Now

$$
\begin{aligned}
& \int_{\text {Shell }(0,0)} f\left(x^{2}\right)|x|_{p} d x=\sum_{i=1}^{p-1} \int_{B(i, 1)} f\left(x^{2}\right)|x|_{p} d x \\
& =\sum_{i=1}^{p-1} \int_{B(i, 1)} 1_{B\left(i^{2}, 1\right)} f\left(x^{2}\right)|x|_{p} d x, \quad \text { by Proposition } 2.1 .
\end{aligned}
$$

But by using Proposition 2.2 and Lemma 2.2 we can show

$$
\int_{B(i, 1)} 1_{B\left(i^{2}, 1\right)} f\left(x^{2}\right)|x|_{p} d x=\int_{B(p-i, 1)} 1_{B\left((p-i)^{2}, 1\right)} f\left(x^{2}\right)|x|_{p} d x
$$

for all $i=1, \ldots,(p-1) / 2$. So we have

$$
\begin{aligned}
\int_{\text {Shell }(0,0)} f\left(x^{2}\right)|x|_{p} d x & =2\left[\sum_{i=1}^{(p-1) / 2} \int_{S(B(i, 1))} 1_{B\left(i^{2}, 1\right)} f(x) d x\right] \\
& =2 \int_{S(\operatorname{Shell}(0,0))} f(x) d x .
\end{aligned}
$$

Proposition 2.3 is also true for general shells. In fact we get the following corollary. 
Corollary. $\int_{\text {Shell }(0,-l)} f\left(x^{2}\right)|x|_{p} d x=2 \int_{S(\text { Shell }(0,-l))} f(x) d x$ for all $f \in C_{c}^{\infty}\left(\mathbf{Q}_{p}\right)$ and for all $l \in \mathbf{Z}$ where $\operatorname{Shell}(0,-l)=\left\{\left.x \in \mathbf{Q}_{p}|| x\right|_{p}=p^{l}\right\}$. This can be proved by using Proposition 2.3 and Lemma 2.2. Although the proof is easy but lengthy, we omit it.

With the dominated convergence theorem, the corollary to Proposition 2.3 and Lemma 2.2 we get the following $p$-adic change of variable formula.

Proposition 2.4. Let $p \neq 2$ be a rational prime number. Then

$$
\int_{\mathbf{Q}_{p}} f\left(x^{2}\right)|x|_{p} d x=2 \int_{S\left(\mathbf{Q}_{p}\right)} f(x) d x
$$

for all $f \in C_{c}^{\infty}\left(\mathbf{Q}_{p}\right)$. Here $S(x)=x^{2}$.

2. Plancherel formula for $N \cdot A$ over a $p$-adic number field. We first need to describe the generic irreducible unitary representation of $N \cdot A$ i.e., those which contribute to the Plancherel formula. The center of $N$ is $Z=\mathbf{Q}_{p}$ (recall we identified $N \cdot A$ with $\mathbf{Q}_{p} \times \mathbf{Q}_{p}^{n} \times \mathbf{Q}_{p}^{n} \times \mathbf{Q}_{p}^{s}$ at the beginning of this chapter). Let $\mathbf{Z}$ be the Lie algebra of $Z$ and $\mathbf{Z}^{s}$ be the dual space of $\mathbf{Z}$ as $\mathbf{Q}_{p}$-vector space.

Now, for each $\lambda \in \mathbf{Z}^{s}$ which is nonzero, there exists an irreducible unitary representation class $\left[\gamma_{\lambda}\right] \in \widehat{N}$, the set of all equivalence classes of irreducible unitary representations of $N$, uniquely determined by the equation $\gamma_{\lambda}(z n)=$ $\lambda(z) \gamma_{\lambda}(n)$ for $z \in Z$ and $n \in N$ (see for details [20, 13, 4]). In fact let $N=$ Lie algebra of $N$. We choose a polarization $\mathbf{U}$ at $\lambda \in \mathbf{N}^{s}$, i.e., $\mathbf{U}$ is a maximal isotropic subspace for the bilinear form $B_{\lambda}$ defined by $\lambda$ as $B_{\lambda}\left(n_{1}, n_{2}\right)=$ $\lambda\left(\left[n_{1}, n_{2}\right]\right)$ for $n_{1}, n_{2} \in \mathbf{N}$. Let $\chi_{\lambda}$ be a character of $U=\exp \mathbf{U}$ by $\chi_{\lambda}(u)=$ $\chi_{0}(\lambda(z))$, where $z$ is the component of $u$ from the center and $\chi_{0}$ is the fixed character of $\mathbf{Q}_{p}$ as usual. Then $\gamma_{\lambda}=\operatorname{Ind}_{U}^{N}\left(\chi_{\lambda}\right)$. We remark that the reason why it is sufficient to consider $\lambda \in \mathbf{Z}^{s}$ instead of $\lambda \in \mathbf{N}^{s}$ is that in our particular case, for any $\lambda \in \mathbf{N}^{s}, \lambda([z, y])=\lambda([z, x])=\lambda\left(\left[x, x^{\prime}\right]\right)=\lambda\left(\left[y, y^{\prime}\right]\right)=0$ for any $z, y, y^{\prime}, x$, and $x^{\prime}$. So we only need to consider $\lambda \in \mathbf{Z}^{s}$ and extend it trivially to $\mathbf{N}$.

We recall that $\left[\gamma_{\lambda}\right] \neq\left[\gamma_{\lambda^{\prime}}\right]$ if $\lambda \neq \lambda^{\prime}$ and these are the generic representation of $N$. The generic representations of $N \cdot A$ are the induced representations induced from those $\left[\gamma_{\lambda}\right]$ such that $\gamma_{\lambda}$ is a representative of $A$-orbit on $\widehat{N} . A$ acts on $\left[\gamma_{\lambda}\right]$ by $a \cdot\left[\gamma_{\lambda}\right]=\left[\gamma_{a^{2} \lambda}\right]$. So we use only four equivalence classes due to the fact that $\mathbf{Q}_{p}^{s}$ has exactly four cosets of the subgroup of elements which are squares of some other elements (see [3]).

$N \cdot A$ has the right Haar measure. We denote it by

$$
d(z, y, x, a)=\frac{1}{|a|_{p}} d x d y d x d a,
$$

where $d z, d y, d x, d a$ are invariant measures on $\mathbf{Q}_{p}, \mathbf{Q}_{p}^{n}, \mathbf{Q}_{p}^{n}$, and $\mathbf{Q}_{p}$ respectively with $d z$ and $d a$ normalized so that the measure of the unit ball is one. 
Theorem 3.1. Denote $\underline{D}=D^{n+1} /{ }^{n+1} \sqrt{2}$, where $D$ is the strong derivative (Chapter $\mathrm{I}$, part $\mathrm{I})$. Let $u$ be a unit element in $\mathbf{Q}_{p}$ that does not represent a square in the residue class field. (For example if $p=3, u=2$, and if $p=5, u$ is either 2 or 3.) Let $\pi_{\lambda}=\operatorname{Ind}_{N}^{N \cdot A}\left(\gamma_{\lambda}\right)$ for $\lambda=1, p, u$, up. Let $E=\left\{\psi \in C_{c}^{\infty}(N \cdot A) \mid \psi\right.$ is a linear combination of $\varphi * \varphi^{s}$ with $\left.\varphi \in C_{c}^{\infty}(N \cdot A)\right\}$. If $\psi \in E$, then $\underline{D} \psi \in L^{1}(N \cdot A)$ and $\pi_{\lambda}(\underline{D} \psi)$ is trace class for $\lambda \neq 0$. The Fourier inversion formula for $N \cdot A$ is

$$
\psi\left(1_{N \cdot A}\right)=T_{1}(\underline{D} \psi)+p T_{p}(\underline{D} \psi)+T_{n}(\underline{D} \psi)+p T_{n p}(\underline{D} \psi)
$$

for all $\psi \in E$, where $T_{\lambda}(\psi)=$ trace of $\pi_{\lambda}(\psi)$.

Comment. The space $E$ is dense in $L^{2}(N \cdot A)$.

Proof of Theorem 3.1. Notice that every element $f$ in the representation space of $\pi_{\lambda}$ can be written as $f(z, y, x, a)=\chi_{\lambda}(z, y, 0,1) f(0,0, x, a)$ because $f(z, y, x, a)=f((z, y, 0,1)(0,0, x, a))=\chi_{\lambda}(z ; y, 0,1) f(0,0, x, a)$. Let $\varphi_{f}(x, a)=f(0,0, x, a)$. Then

$$
\varphi_{f} \in L^{2}\left(\mathbf{Q}_{p}^{n} \times \mathbf{Q}_{p}^{s}, \frac{1}{|a|_{p}} d x d a\right) .
$$

Moreover, the map

$$
L^{2}\left(\frac{N \cdot A}{U}, \chi_{\lambda}\right) \rightarrow L^{2}\left(\mathbf{Q}_{p}^{n} \times \mathbf{Q}_{p}^{s}, \frac{1}{|a|_{p}} d x d a\right)
$$

by $f \mapsto \varphi_{f}$ is unitary and intertwines $\pi_{\lambda}$ and $\tilde{\pi}_{\lambda}$, where

$$
\tilde{\pi}_{\lambda}(z, y, x, a): L^{2}\left(\mathbf{Q}_{p}^{n} \times \mathbf{Q}_{p}^{s}, \frac{1}{|a|_{p}} d x d a\right) \rightarrow L^{2}\left(\mathbf{Q}_{p}^{n} \times \mathbf{Q}_{p}^{s}, \frac{1}{|a|_{p}} d x d a\right)
$$

is defined by $\tilde{\pi}_{\lambda}(z, y, x, a) \varphi_{f}=\varphi_{\pi_{\lambda}(z, y, x, a) f}$. So we view $\pi_{\lambda}$ as a representation on

$$
L^{2}\left(\mathbf{Q}_{p}^{n} \times \mathbf{Q}_{p}^{s}, \frac{1}{|a|_{p}} d x d a\right) .
$$

We use the same notation $\pi_{\lambda}$ instead of $\tilde{\pi}_{\lambda}$. Then

$$
\begin{aligned}
& \pi_{\lambda}\left(\left(z^{\prime}, y^{\prime}, x^{\prime}, a^{\prime}\right) \varphi_{f}\right)(x, a)=f\left(a^{2} z^{\prime}+a x \cdot y^{\prime}, a y^{\prime}, x+a x^{\prime}, a a^{\prime}\right) \\
& \quad=f\left(\left(a^{2} z^{\prime}+a^{2} x^{\prime} \cdot y^{\prime}+2 a x \cdot y^{\prime}, a y^{\prime}, 0,1\right)\left(0,0, x+a x^{\prime}, a a^{\prime}\right)\right) \\
& \quad=\chi_{\lambda}\left(a^{2} z^{\prime}+a^{2} x^{\prime} \cdot y^{\prime}+2 a x \cdot y^{\prime}\right) \varphi_{f}\left(x+a x^{\prime}, a a^{\prime}\right) .
\end{aligned}
$$


Now let $\psi \in C_{c}^{\infty}(N \cdot A)$. Then

$$
\begin{aligned}
& \left(\pi_{\lambda}(\psi) \varphi_{f}\right)(x, a) \\
& =\int_{N \cdot A} \psi\left(z^{\prime}, y^{\prime}, x^{\prime}, a^{\prime}\right)\left(\pi_{\lambda}\left(z^{\prime}, y^{\prime}, x^{\prime}, a^{\prime}\right) \varphi_{f}\right)(x, a) \frac{1}{\left|a^{\prime}\right|_{p}} d z^{\prime} d y^{\prime} d x^{\prime} d a^{\prime} \\
& =\int_{N \cdot A} \psi\left(z^{\prime}, y^{\prime}, x^{\prime}, a^{\prime}\right) \chi_{\lambda}\left(a^{2} z^{\prime}+a^{2} x^{\prime} \cdot y^{\prime}+2 a x \cdot y^{\prime}\right) \\
& \cdot \varphi_{f}\left(x+a x^{\prime}, a a^{\prime}\right) \frac{1}{\left|a^{\prime}\right|_{p}} d z^{\prime} d y^{\prime} d x^{\prime} d a^{\prime} .
\end{aligned}
$$

We claim that

$$
\begin{aligned}
& \left(\pi_{\lambda}(\psi) \varphi_{f}\right)(x, a) \\
& =\int_{\mathbf{Q}_{p}^{n} \times \mathbf{Q}_{p}^{s}} \hat{\psi}\left(\cdot, \cdot, \frac{x^{\prime}-x}{a}, \frac{a^{\prime}}{a}\right)\left(\lambda a^{2}, \lambda a\left(x^{\prime}+x\right)\right) \varphi_{f}\left(x^{\prime}, a^{\prime}\right)\left|a^{\prime}\right|_{p}^{-n} \frac{1}{\left|a^{\prime}\right|_{p}} d x^{\prime} d a^{\prime}
\end{aligned}
$$

where $\hat{\psi}$ is the Fourier transform of $\psi$ in the first two variables.

Now let

$$
K\left(x, a, x^{\prime}, a^{\prime}\right)=\hat{\psi}\left(\cdot, \cdot \frac{x^{\prime}-x}{a}, \frac{a^{\prime}}{a}\right)\left(\lambda a^{2}, \lambda a\left(x^{\prime}+x\right)\right) \frac{|a|_{p}^{-n}}{\left|a^{\prime}\right|_{p}} .
$$

Then

$$
\left(\pi_{\lambda}(\psi) \varphi_{f}\right)(x, a)=\int_{\mathbf{Q}_{p}^{n} \times \mathbf{Q}_{p}^{s}} K\left(x, a, \frac{x^{\prime}-x}{a}, a^{\prime}\right) \varphi_{f}\left(x^{\prime}, a^{\prime}\right) d x^{\prime} d a^{\prime} .
$$

Now if $\psi \in E$ in particular, $\pi_{\lambda}(\psi)$ is of trace class. Let $T_{\lambda}(\psi)=$ trace of $\pi_{\lambda}(\psi)$. Then

$$
\begin{aligned}
T_{\lambda}(\psi) & =\int_{\mathbf{Q}_{p}^{n} \times \mathbf{Q}_{p}^{s}} K(x, a, \lambda, a) d x d a \\
& =\int_{\mathbf{Q}_{p}^{n} \times \mathbf{Q}_{p}^{s}} \hat{\psi}(\cdot, \cdot, 0,1)\left(\lambda a^{2}, 2 \lambda a x\right)|a|_{p}^{-(n+1)} d x d a \\
& =\int_{\mathbf{Q}_{p}^{n} \times \mathbf{Q}_{p}^{s}} \hat{\psi}(\cdot, \cdot, 0,1)\left(\lambda a^{2}, x\right)|a|_{p}^{-(n+1)} \frac{|x|_{p}^{n}}{|2 \lambda a|_{p}^{n}}=\frac{1}{|x|_{p}} d x d a \\
& =\int_{\mathbf{Q}_{p}^{n} \times \mathbf{Q}_{p}^{s}} \hat{\psi}(\cdot, \cdot, 0,1)\left(\lambda a^{2}, x\right) \frac{1}{|\lambda|_{p}^{n}}|a|_{p}^{-(2 n+1)} d x d a .
\end{aligned}
$$

We let $g: \mathbf{Q}_{p} \rightarrow \mathbf{C}$ be defined by $g(v)=\hat{\psi}(\cdot, \cdot, 0,1)(\lambda v, x)|v|_{p}^{-(n+1)}$. Then $g \in C_{c}^{\infty}(\mathbf{Q})$ for each fixed $x$. Thus using Proposition 2.4

$$
\begin{aligned}
T_{\lambda}(\psi) & =\int_{\mathbf{Q}_{p}^{n}} \int_{\mathbf{Q}_{p}^{s}} g\left(a^{2}\right)|a|_{p} \frac{1}{|\lambda|_{p}^{n}} d a d x \\
& =\frac{2}{|\lambda|_{p}^{n}} \int_{\mathbf{Q}_{p}^{n}} \int_{S\left(\mathbf{Q}_{p}^{s}\right)} \hat{\psi}(\cdot, \cdot, 0,1)(\lambda a, x)|a|_{p}^{-(n+1)} d a d x .
\end{aligned}
$$


Now,

$$
\begin{aligned}
& \psi\left(1_{N \cdot A}\right)=\psi(0,0,0,1)=\int_{\mathbf{Q}_{p}^{s}} \int_{\mathbf{Q}_{p}^{n}} \psi(\cdot, \cdot, 0,1) \widehat{(a, x) d a d x} \\
& =\int_{S\left(\mathbf{Q}_{p}^{s}\right)} \int_{\mathbf{Q}_{p}^{n}} \psi(\cdot, \cdot, 0,1) \widehat{(a, x) d a d x} \\
& +\int_{S\left(\mathbf{Q}_{p}^{s}\right)} \int_{\mathbf{Q}_{p}^{n}} \psi(\cdot, \cdot, 0,1)^{\wedge}(a p, x) d a d x \\
& +\int_{S\left(\mathbf{Q}_{p}^{s}\right)} \int_{\mathbf{Q}_{p}^{n}} \psi(\cdot, \cdot, 0,1)^{\wedge}(a u, x) d a d x \\
& +\int_{S\left(\mathbf{Q}_{p}^{s}\right)} \int_{\mathbf{Q}_{p}^{n}} \psi(\cdot, \cdot, 0,1) \wedge(\operatorname{aup}, x) d a d x \\
& =\int_{S\left(\mathbf{Q}_{p}^{s}\right)} \int_{\mathbf{Q}_{p}^{n}} D^{n+1} \psi(\cdot, \cdot, 0,1) \uparrow(a, x)|a|_{p}^{-(n+1)} d a d x \\
& +\int_{S\left(\mathbf{Q}_{p}^{s}\right)} \int_{\mathbf{Q}_{p}^{n}} D^{n+1} \psi(\cdot, \cdot, 0,1)^{-}(a p, x)|a p|_{p}^{-(n+1)} d a d x \\
& +\int_{S\left(\mathbf{Q}_{p}^{s}\right)} \int_{\mathbf{Q}_{p}^{n}} D^{n+1} \psi(\cdot, \cdot, 0,1) \uparrow(a u, x)|a u|_{p}^{-(n+1)} d a d x \\
& +\int_{S\left(\mathbf{Q}_{p}^{s}\right)} \int_{\mathbf{Q}_{p}^{n}} D^{n+1} \psi(\cdot, \cdot, 0,1)^{-}(\operatorname{aup}, x)|a u p|_{p}^{-(n+1)} d a d x \\
& =\frac{1}{2} \operatorname{tr} \pi_{1}\left(D^{n+1} \psi\right)+\frac{p}{2} \operatorname{tr} \pi_{p}\left(D^{n+1} \psi\right) \\
& +\frac{1}{2} \operatorname{tr} \pi_{n}\left(D^{n+1} \psi\right)+\frac{p}{2} \operatorname{tr} \pi_{s p}\left(D^{n+1} \psi\right) \\
& =T_{1}(\underline{D} \psi)+p T_{p}(\underline{D} \psi)+T_{n}(\underline{D} \psi)+p T_{p}(\underline{D} \psi) \text {. }
\end{aligned}
$$

Acknowledgement. This is a part of the author's doctoral dissertation. She wishes to thank Professor Joseph A. Wolf for his advice and encouragement.

\section{REFERENCES}

1. J. Dixmier, Algébres quasi-unitaries, Comment. Math. Helv. 26 (1952), 275-322.

2. M. Duflo and C. C. Moore, On the regular representation of a non-unimodular locally compact group, J. Funct. Anal. 21 (1976), 209-243.

3. I. M. Gel ' fand, M. I. Graev, and I. I. Pyatetskii-Shapiro, Representation theory and automorphic functions, Saunders, Philadelphia, Pa., 1969.

4. F. W. Keene, Square integrable representations and Plancherel theorem for parabolic groups, Trans. Amer. Math. Soc. 243 (1978), 61-73.

5. F. W. Keene, R. L. Lipsman, and J. A. Wolf, The Plancherel formula for parabolic subgroups, Israel J. Math. 28 (1977), 68-90.

6. A. A. Kirillov, Unitary representations of nilpotent Lie groups, English transl., Russian Math. Surveys 17 (1962), no. 4, 53-104.

7. A. Kleppner and R. L. Lipsman, The Plancherel formula for group extensions, Ann. Sci. Ecole Norm. Sup. 5 (1972), 459-516. 
8. - The Plancherel formula for group extensions. II, Ann. Sci. Ecole Norm. Sup. 6 (1973), 103-132.

9. T. Y. Lam, The algebraic theory of quadratic forms, Benjamin, 1973.

10. R. L. Lipsman and J. A. Wolf, The Plancherel formula for parabolic subgroups of the classical groups, Trans. Amer. Math. Soc. 260 (1980), 607-622.

11. _ Canonical semi-invariants and the Plancherel formula for parabolic groups, Trans. Amer. Math. Soc. 269 (1982), 111-131.

12. C. C. Moore, Decomposition of unitary representations defined by discrete subgroups of nilpotent groups, Ann. of Math. 82 (1965), 146-182.

13. __ A Plancherel formula for non-unimodular groups, Address presented to the Internat. Conf. on Harmonic Analysis, Univ. of Maryland, 1971.

14. __ Representations of solvable and nilpotent groups and harmonic analysis on nil and solomanifolds, Proc. Sympos. Pure Math., vol. 26, Amer. Math. Soc., Providence, R. I., 1973, pp. 3-44.

15. M. Nakata, Harmonic analysis on local fields, Doctoral Dissertation, Univ. of California, Berkeley, 1983.

16. C. W. Onneweer, On the definition of dyadic differentiation, Department of Math. and Stat, Univ. of New Mexico, Tech. Rep. No. 344 (1978).

17. L. Pukanszky, On the theory of quasi-unitary algebras, Acta Sci. Math. 16 (1955), 103-121.

18. I. Satake, Classification of semi-simple algebraic groups, Dekker, 1971.

19. M. H. Taibleson, Fourier analysis on local fields, Math. Notes, Princeton University Press, 1975.

20. N. Tatsuuma, Plancharel formula for non-unimodular locally compact groups, J. Math. Kyoto Univ. 12 (1972), 179-261.

21. G. van Dijk, Smooth and admissible representations of p-adic unipotent groups, Compositio Math. 37 (1978), 77-101.

22. J. A. Wolf, Fourier inversion problems on Lie groups and a class of pseudo-differential operators, Conf. Partial Differential Equations and Geometry, Park City, Utah, 1977.

23. __ Unitary representations of maximal parabolic subgroup of the classical group, Mem. Amer. Math. Soc., vol. 8, no. 180 (1976).

24. E. Weiss, Algebraic number theory, McGraw-Hill, 1963.

Department of Mathematical Sciences, Rice University, Houston, Texas 77251

Current address: D-1101 Kamishinden 4-8, Toyonaka, Osaka 565, Japan 